\title{
URINARY INCONTINENCE
}

\section{The bladder sets its own clock}

Patients with bladder dysfunction, such as overactive bladder, often have altered circadian urinary rhythms and unwanted nocturnal urgency. Now, Changhao Wu and colleagues from the University of Surrey, UK, have shown that expression of the 'clock protein' period circadian protein homolog 2 (PER2) in the bladder wall follows a diurnal rhythm and determines circadian bladder contractility. Moreover, this process is regulated locally by physiological and pathological signals and its functionality is influenced by bladder age.

\section{4 ...loss of normal circadian control ... favours unwanted bladder muscle activity... 77}

In their study published in The FASEB Journal, the researchers used bladder samples from Per2::Luc knock-in mice, which exhibit PER2 expression patterns, circadian entrainment and behaviours that are similar to their wild-type littermates.

"This model enables real-time, continuous recording of the clock activities in live tissue with physiological relevance," Wu explains. "In addition, we used fullthickness bladder wall specimens with intact mucosa and smooth muscle to maintain the integrity of the physiology." Both the urothelial mucosa and smooth muscle expressed PER2 following a diurnal rhythm, with peak expression (more than threefold increase in luciferase activity) at $\sim 12 \mathrm{~h}$.
Next, the researchers stimulated muscarinic and purinergic receptors with carbachol and ATP- $\gamma-S$, respectively, and found that the peak in PER2 expression occurred $\sim 5$ h earlier than without stimulation-demonstrating that clock activity is locally regulated. Of note, mild mechanical stress significantly increased PER2 levels, highlighting the influential role of this stimulus in visceral organs.

"We also measured the contractility of the bladder specimens to demonstrate the functional importance of the circadian rhythms," Wu points out. Contractile activity stimulated by muscarinic activation followed the pattern of normal PER2 expression with a peak in contractile force at $\sim 12 \mathrm{~h}$, whereas muscle contractions induced by electrical-field stimulation did not show clock dependence. These results suggest that local receptor expression in the bladder wall regulates muscle contractility in a circadian fashion.

$\mathrm{Wu}$ and colleagues also investigated whether this regulatory system was subject to age-related changes: peak PER2 expression decreased with increasing age of evaluated bladder specimens (range 1-12 months). Importantly, the temporal shift in PER2 expression observed after muscarinic and purinergic stimulation in samples from 3-5 month-old mice was absent in samples from 9-12 month-old mice, pointing towards an impairment in the local circadian control system in bladders from old mice.

"Our study helps in understanding how the local clocks in the bladder are

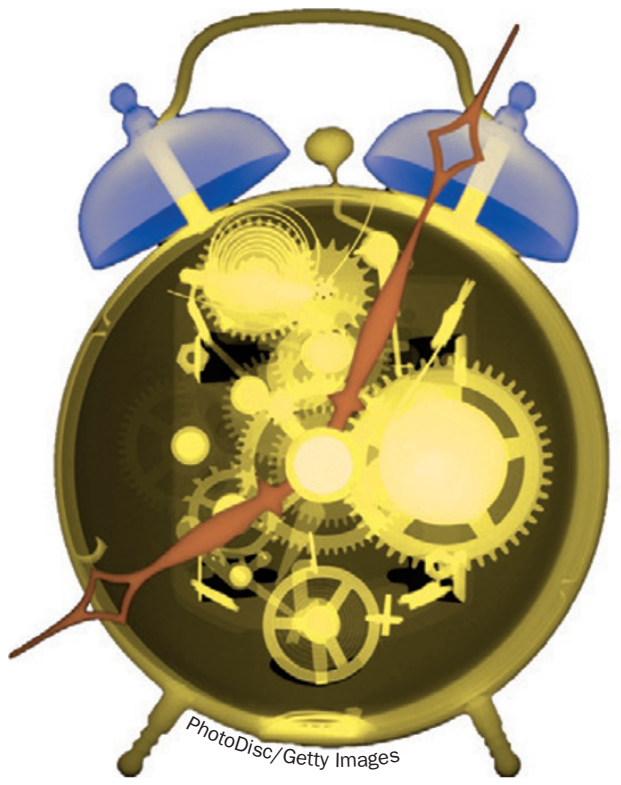

regulated and provides unequivocal evidence for the rhythmicity of the smooth muscle tissue of the bladder," Wu summarizes. "We hypothesize that, in 'aged' bladders, the loss of normal circadian control and, hence, intrinsic rhythmicity favours unwanted bladder muscle activity, such as involuntary contractions and nocturnal bladder overactivity." The researchers hope that their findings will aid the development of new treatments for altered urinary activity based on strategies that target this newly discovered circadian regulatory system.

\section{Clemens Thoma}

Original article Wu, C. et al. Local receptors as novel regulators for peripheral clock expression. FASEB J. doi:10.1096/fj.13-243295 